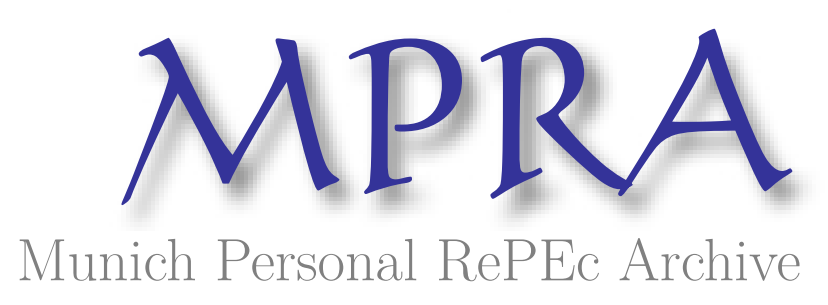

\title{
Outsourcing and Vertical Integration in a Competitive Industry
}

Ciliberto, Federico and Panzar, John

30 October 2009

Online at https://mpra.ub.uni-muenchen.de/38628/

MPRA Paper No. 38628, posted 07 May 2012 14:45 UTC 


\title{
Outsourcing and Vertical Integration in a Competitive Industry*
}

\author{
Federico Ciliberto ${ }^{\dagger}$ and John C. Panzar \\ October 2009 \\ Forthcoming in the Southern Economic Journal.
}

\begin{abstract}
We develop a partial equilibrium, perfectly competitive framework of a (potentially) vertically integrated industry. There are three types of firms: upstream firms that use primary factors to produce an intermediate; downstream firms that use primary factors and intermediates to produce a final good; and vertically integrated firms that do both. We establish conditions under which vertically integrated firms exist and outsource (part of) the production of the intermediate input. We study the changes in industry configurations resulting from changes in costs and demand.
\end{abstract}

JEL Classification: F11, L11, L22.

Keywords: Competitive Industry, Vertical Integration, Outsourcing.

\footnotetext{
*We would like to thank two referees and the Editor for their comments, as well as seminar participants at the 2001 European Association for Research in Industrial Economics Conference in Dublin, at the 2001 Northeast Universities Development Consortium Conference Conference in Boston, and at the 2001 Southeastern Economic Theory and International Trade Conference in Miami. Federico Ciliberto acknowledges financial support from the Center for the Studies of Industrial Organization at Northwestern University. This paper is revised version of the third chapter of the PhD Dissertation of Federico Ciliberto at Northwestern University.

†Department of Economics, University of Virginia, ciliberto@virginia.edu, ph: 434-924-6755.

†The University of Auckland Business School, j.panzar@auckland.ac.nz, ph: +64-93737599.
} 


\section{Introduction}

We develop a partial equilibrium, competitive framework of a (potentially) vertically integrated industry. In our model there are three types of firms: upstream firms that use primary factors to produce an intermediate; downstream firms that use primary factors and intermediates to produce a final good; and vertically integrated firms that do both. We establish conditions under which vertically integrated firms exist and outsource (part of) the production of the intermediate input. Specifically, we ask the following questions: Why do competitive firms vertically integrate? What changes in the economy, and, in particular, in the demands of the intermediate and final goods, can explain the vertical disintegration or integration of competitive industries?

We build on the literature of multiproduct firms in competitive markets. That literature illustrates market structures when there are two final goods: A and B. This paper uses a very similar framework, except the distinction being that good B is intermediate good in the production of A. The results hinge on the concept of economies of scope. The comparative statics show how the industry structure changes in response to changes in the external demand of final goods and intermediate goods and also changes in the cost structure. In particular, we show that if economies of vertical scope are present then the vertical organization of the industry is determined by the relative ratio of demands of intermediate and final output and by the ratio of costs. As the demand external to the industry for the intermediate output changes, the equilibrium configuration of the industry changes as well. To develop our analysis we use the simplest functions forms for cost and demand that are necessary to demonstrate the results. We then justify how our results carry forward under more general forms. As such, we hope that our simplifications are considered as a point of strength rather than a point of critique.

This paper formalizes George Stigler's [1951] take on vertical integration in his "The division of Labor is Limited by the Extent of the Market." Stigler shows that when the industry grows, the vertically integrated firm outsources (part of) the production of the intermediate good whose production process is subject to increasing costs. Stigler also shows that when one of the production processes displays increasing returns to scale, it will be turned over to specialists as the market grows. The specialists cannot charge more than the average incremental cost that the vertically integrated firm would face if it were producing 
the intermediate input in-house. Thus the specialists face a negatively sloped elastic demand for the intermediate input. As the market continues to grow, the new industry will become competitive. Stigler limits the analysis to the case in which there are no economies of vertical scope and one of the production processes is subject to increasing costs.

To formalize George Stigler's [1951] take on vertical integration we extend the competitive framework developed by MacDonald and Slivinski [1987]. First, we allow for economies of vertical scope to exist and we study when outsourcing occurs in the industry. In our model (partial) outsourcing of the production plays a critical role in determining which firms are present in equilibrium. Clearly, there is no scope for outsourcing in MacDonald and Slivinski's horizontal multiproduct industry. Second, we determine the set of output combinations that vertically competitive firms choose in equilibrium. From this set of output combinations we are able to conclude whether there are only vertically integrated firms in the industry, or whether there are also upstream or downstream firms. Third, we study the industry when another industry sells the intermediate good into the market. We show how the vertical structure of the industry depends on the ability of the producers of intermediate output to compete at lower prices than the upstream firms.

Before moving on to the analysis, we want to stress how our model explains vertical integration patterns from a technological perspective. In this sense, our approach stands in contrast to the incomplete contracting literature (Williamson [1985], Hart and Moore [1988]) which often dismisses technological explanations of vertically integration.

In section 2 of the paper we introduce the notions of economies of vertical scope. In section 3 we introduce the notion of economies of scope. In section 4 we define a vertical competitive equilibrium. In section 4 we study the vertical structure and equilibrium of the industry when vertically integrated firms cannot outsource the production of the intermediate input and when the industry produces a surplus of the intermediate output. In section 5 of the paper we still consider the industry as producing a surplus of the intermediate input but the vertically integrated firms now outsource the production of the input. In section 6 we merge the results from section 3 and 4, and we present a comparative statics analysis to show how the industry structure changes with changes in the aggregate output demands. Finally, in section 7 we present the case when the industry buys the intermediate input. Section 8 summarizes the results and concludes the paper. 


\section{Economies of Specialization and of Vertical Scope}

The industry deals with three categories of commodities: a final good $y \geq 0$, an intermediate product $k$, and a vector of primary factors $\mathbf{x} \leq \mathbf{0}$. This netput notation helps facilitate the analysis. Let $p, r$, and $\mathbf{w}$ denote the associated market prices. Firms are price takers in all markets and there is free entry in the markets of the final good. There is free entry in the market of the intermediate product when it is an output. $p^{e}$ denote the equilibrium price of the final good. $r^{e}$ denotes the equilibrium price of the intermediate product when it is an output.

The firm is upstream if it only produces $k$, and it is downstream if it only produces $y$. For any firm under study, the final good is always an output $(y \geq 0)$ and the primary factors are always inputs $(\mathbf{x} \leq \mathbf{0})$. However, the intermediate product is always an output

for upstream firms $\left(k^{U} \geq 0\right)$ and an input for downstream firms $\left(k^{D} \leq 0\right)$. The net supply of the intermediate product for a vertically integrated firm may be positive, negative, or zero. That is, an integrated firm may operate a process that generates more, less, or exactly the amount of the intermediate product that it requires to produce a specified level of the final good.

More formally, firms are assumed to operate one of the three production sets:

$$
\begin{aligned}
Y_{U} & =\left\{(\mathbf{x}, y, k) \in \Re^{m+2} \mid 0 \leq k \leq f(-\mathbf{x}) ; y=0, \mathbf{x} \leq \mathbf{0}\right\}, \\
Y_{D} & =\left\{(\mathbf{x}, y, k) \in \Re^{m+2} \mid 0 \leq y \leq g(-\mathbf{x},-\mathbf{k}) ; k \leq 0, \mathbf{x} \leq \mathbf{0}\right\} \\
Y_{I} & =\left\{(\mathbf{x}, y, k) \in \Re^{m+2} \mid H(x, y, k) \leq 0 ; y \geq 0, \mathbf{x} \leq \mathbf{0}\right\} .
\end{aligned}
$$

In this formulation, $f$ and $g$ are traditional production functions and $H$ is a production transformation function.

Next, we need to develop definitions that reflect intuitive definitions of the technological advantages of specialization and integration. First, we consider the production of the intermediate good. The notion of specialization in the production is related to the ability of firms of producing one single good more efficiently than multiproduct firms can do if they only produce the same good. The following two definitions capture this notion of specialization:

Definition 1 Economies of Upstream Specialization. When none of the final good 
is produced, the upstream technology is at least as good as the integrated technology. That is, $Y_{U} \supseteq Y_{I}$ for $y=0$. Equivalently, this means that $f(-x) \geq$ $\max _{k}\{k \mid H(\mathbf{x}, 0, k) \leq 0\}$.

Definition 2 Economies of Downstream Specialization. When the intermediate good is not an output, the downstream technology is at least as good as the integrated technology. That is, $Y_{D} \supseteq Y_{I}$ for $k \leq 0$.

Equivalently, $g(-\mathbf{x},-k) \geq \max _{y}\{y \mid H(\mathbf{x}, y, k) \leq 0, k \leq 0\}$.

The notion of downstream specialization is less intuitive than that of upstream specialization. To see why, observe that only in exceptional cases the minimum efficient scale of producing an intermediate is proportional to that of producing the final good, so that the technologies are perfectly matched in terms of their outputs. Hence, a vertically integrated firm normally produces too much or too little of the intermediate output for its own use. This is why we expect economies of downstream specialization to exist.

When the vertically integrated firm can sell the intermediate good as an output, then it can fully exploit economies of scope in the production of the final and intermediate goods. We define economies of vertical scope next:

Definition 3 Economies of Vertical Scope. A collection of upstream, downstream, and integrated technologies are said to exhibit economies of vertical scope if and only if $Y_{I} \supseteq Y_{U}+Y_{D}$. Equivalently, given $k^{U} \geq 0, k^{D} \leq 0, \mathbf{x}^{U} \leq 0, \mathbf{x}^{D} \leq 0$ and $y^{D} \geq 0$ such that $k_{U} \leq f\left(-\mathbf{x}^{U}\right)$ and $y^{D} \leq g\left(-\mathbf{x}^{D},-k^{D}\right)$, then $H\left(\mathbf{x}^{U}+\mathbf{x}^{D}, y^{D}, k^{U}+k^{D}\right) \leq 0$.

We expect to observe economies of vertical scope when producing a final good lowers the costs of producing the intermediate output. For example, if learning by doing is important in designing the best intermediate input for the final good, then producing them together can be cheaper than producing them separately. However, if the intermediate and final goods are standardized, then economies of vertical scope are less likely to exist.

\section{Vertical Competitive Equilibrium}

There are two questions we need to address: First, are there vertically integrated firms in the vertical competitive equilibrium? Second, can there be both vertically integrated and 
specialized firms in the equilibrium? The first questions is easily answered. Panzar and Willig's [1981] result that economies of scope are necessary and sufficient for the existence of multiproduct firms in equilibrium is still valid here. ${ }^{1}$ Thus, we need to address the second question, whether there are also upstream and downstream firms in equilibrium, and we need to determine conditions that explain which type of firm is present in equilibrium.

To address this question, we follow MacDonald and Slivinski [1987] and introduce some structure in the model. We denote the aggregate demand of final output by $Y$ and the net demand of the intermediate input by $K$. If $K>0$, then $K$ is sold (we might say "exported" to make it simpler to understand) to another industry which uses the same intermediate input. If $K<0$, then $K$ is bought ("imported") from another industry.

We assume that $K$ and $Y$ are exogenous to simplify the analysis. ${ }^{2}$ However, we could assume that $Y(p)=z(c-d p)$ and $K=z(a-b r)$, where $z$ is a large positive integer, and the results of this paper would still carry on. This latter weaker assumption would ensure that there is always an interior solution where all firms of the same type produce the same combination of outputs. The prices $p$ and $k$ are still endogenous.

Let $k_{p}$ be the amount of intermediate input that the vertically integrated firm purchases on the market, and let $k_{i}$ be the amount of intermediate output that the vertically integrated firm produces for its own use. Then $k_{i}+k_{p}=k(y)$, where $k(y)$ is the total amount of $k$ that the vertically integrated firm needs to produce $y$. We define by $k_{s}$ the surplus of intermediate input that the vertically integrated firm may produce. We define by $k^{U}$ the amount of intermediate output produced by the specialized upstream firms.

We define by $y^{D}$ the amount of final output produced by the specialized downstream firms.

\footnotetext{
${ }^{1}$ Proposition 1 in Panzar and Willig [1981] states that economies of scope are sufficient for the existence of multiproduct firms in a multiproduct competitive equilibrium, and weak economies of scope are necessary for such existence of a multiproduct firm. Notice that their proposition does not refer to the nature (e.g. final or intermediate) of the goods. Here, a multi-product firm is a vertically integrated firm.

${ }^{2}$ See Eaton and Lemche [1991] for an extension of MacDonald and Slivinski [1987] that allows for endogenous demands. The analysis could be developed with inverse demand schedules $r=A-B K^{s}$ and $P=C-D Y$ instead of a fixed demands $K_{s}^{D}$ and $Y^{D}$. Consider the case when only vertically integrated firms are in the market. Then they would choose $2 w\left(y^{I}+k_{s}^{I}\right)=A-B n^{I} k_{s}^{I}$ and $2 w\left(y^{I}+k_{s}^{I}\right)+2 w y^{I}=C-D n^{I} y^{I}$. Once we add the zero profit condition $\left(F^{I}=w\left(y^{I}+k_{s}^{I}\right)^{2}+w\left(y^{I}\right)^{2}\right)$, these are three equations in three unknowns $\left(y^{I}, k_{s}^{I}\right.$ and $\left.n^{I}\right)$ and the analysis is analogous to the one in the text. When we do the comparative statics exercises, we would study changes in the parameters $A, B, C$ and $D$ rather than $K_{s}^{D}$. For sake of simplicity we have decided to present the model with $K_{s}^{D}$ exogenous. See Baumol, Panzar, and Willig [1982] for more on this.
} 
Let $n^{I}, n^{D}$, and $n^{U}$ denote the number of vertically integrated, downstream, and upstream firms, respectively. Next, we define a competitive vertical equilibrium: ${ }^{3}$

Definition 4 A competitive vertical equilibrium with free entry is given by a pair of prices for the final and intermediate goods $\left(p^{e}, r^{e}\right)$ and two market clearing conditions $Y=$ $n^{I} y^{I}+n^{D} y^{D}$ and $K=\left(k_{s}^{I}-k_{p}^{I}\right) n^{I}+k^{U} n^{U}$, such that the pattern of integration of an industry satisfies the following conditions: i) no active firm can benefit from altering its choice of the stages at which it operates; ii) no active firm can profit by changing output levels; iii) no potential firm finds profitable to enter into the industry; iv) all the firms in the industry, both incumbent and potential entrants, are price takers.

\section{Equilibrium without Outsourcing}

For sake of simplicity of exposition, we assume that the vertically integrated firm cannot buy intermediate input on the market. In section 5 we relax this assumption and let the vertically integrated firm buy some of the intermediate input on the market (this is what we call outsourcing) and study how the comparative statics results change.

In this section we also maintain that $K_{s}>0$, that is the industry produces a surplus of intermediate outputs. The industry sells its surplus of intermediates to another industry.

Following MacDonald and Slivinski [1987], we assume that $k=g(x)=\sqrt{x}$, and $y=$ $\min \left\{\sqrt{x_{y}}, k\right\}$. Hence, $k_{i}+k_{p}=y$. Downstream, upstream and vertically integrated firms face fixed costs equal to $F^{D}, F^{U}$, and $F^{I}$. ${ }^{4}$ Then we can write their cost functions as follows:

\footnotetext{
${ }^{3}$ Perry [1989] first defined a vertical equilibrium to be a pattern of integration in the industry such that no firm would alter its choice of the stages at which it operates. Perry also provides a superb review of the early literature on vertical integration.

${ }^{4}$ The analysis in this paper, as in MacDonald and Slivinski [1987] assumes that the source of economies of scope are differences in the fixed costs across firms. However, we could develop the analysis assuming that there are cost complementarities in the production and no differences in the fixed costs. For example, assume $C^{I_{s}}\left(y, k_{s} ; w\right)=F^{I}+w\left[y(1-d)+k_{s}\right]^{2}+w y^{2}$. The term $d$ denotes the extent to which there are cost complementarities in the production of $y$ and $k$. In particular, observe that $C_{k y}^{I_{s}}\left(y, k_{s} ; w\right)=2 w(1-d)$. If $d>1$ there are cost complementarities in the production of the final and intermediate output. The vertically integrated firm saves money even if $0<d<1$, though there are cost rivalries in such a case. The equilibrium analysis would be analagous, but the results would depend on the magnitude of $d$ rather than on the relationship between $F^{U}, F^{D}$ and $F^{I}$. Interestingly, Stigler [1951] talks about complementary and rival production processes in his article.
} 


$$
\begin{aligned}
C^{U}(k ; w) & =F^{U}+w k^{2}, \\
C^{D}(y ; w, r) & =F^{D}+r y+w y^{2}, \\
C^{I_{s}}\left(y, y+k_{s} ; w, r\right) & =F^{I}+w\left(y+k_{s}\right)^{2}+w y^{2} .
\end{aligned}
$$

The only interesting case is when $F^{I}<F^{U}+F^{D}, F^{U}<F^{I}$, and $F^{D}<F^{I}$. If $F^{I}>F^{U}+F^{D}$, then there are no economies of scope and only specialized firms will be present in equilibrium. To see why, observe that there are marginal rivalries (as opposed to complementarities) between the production of $y$ and $k$ in a vertically integrated firm, which are captured by the interaction term $2 w y k_{s}$. It is the trade-off between marginal rivalries and economies of scope in the fixed costs that leads to the simultaneous presence of vertically integrated and specialized firms. This is the critical element of the paper and does not depend on the functional forms we use and on the fact that we limit the analysis to economies of scope only in the fixed costs. One could envisage a situation where economies of scope are in the marginal costs (i.e. cost complementarities), while rivalries are in the fixed costs. As long as there exists a trade-off, then more than one type of firm can exist in equilibrium.

\subsubsection{The Equilibrium Conditions}

If downstream and upstream firms are present in equilibrium, then the prices of the intermediate input $k$ and of the final output $y$ are uniquely pinned down, and given by $r^{e}=2 \sqrt{F^{U} w}$ and $p^{e}=2 \sqrt{F^{U} w}+2 \sqrt{F^{D} w}$. These are the average costs faced by the specialized firms at

their minimum efficient scales. $k^{\text {mes }}=\sqrt{\frac{F^{U}}{w}}$ and $y^{\text {mes }}=\sqrt{\frac{F^{D}}{w}}$ are the minimum efficient scale outputs for the upstream and downstream firms. Since the industry is perfectly competitive, firms will charge prices where their average costs are the lowest. We now lay out the conditions under which a vertically integrated firm exists.

Vertically integrated firms cannot charge higher prices than the vertically specialized firms in a perfectly competitive industry. Thus $r^{e} \leq 2 \sqrt{F^{U} w}$ and $p^{e} \leq 2 \sqrt{F^{U} w}+2 \sqrt{F^{D} w}$. Moreover, competitive vertically integrated firms must produce locally at constant multiproduct returns to scale otherwise they could increase their profits with a marginal increase or decrease of their production outputs. Hence, the following must hold

$$
C R S: F^{I}=w\left(y^{I}+k_{s}^{I}\right)^{2}+w\left(y^{I}\right)^{2},
$$


Competitive vertically integrated firms must also choose production outputs where the product-specific economies of scale display either increasing or constant returns to scale otherwise they would be facing decreasing returns to scale, and have an incentive to lower their output production. Hence,

$$
S P E C: y^{I} \geq \sqrt{\frac{F^{I}-F^{U}}{2 w}}
$$

Finally, the cost for a vertically integrated firm to produce a combination of outputs must not be larger than those that specialized firms jointly face if they produce at their minimum efficient scale otherwise the specialized firms would be able to charge lower prices than the vertically integrated firm can afford. Hence,

$$
I N T: 2\left(\sqrt{F^{U} w}+\sqrt{F^{D} w}\right) y^{I}+2 \sqrt{F^{U} w} k_{s}^{I} \geq F^{I}+w\left(y^{I}+k_{s}^{I}\right)^{2}+w\left(y^{I}\right)^{2},
$$

These three conditions are very intuitive, and lead to clear comparative statics predictions that depend on $F^{U}, F^{D}$ and $F^{I}$, as shown in Figure 1.

The curve $C R S$ in Figure 1 corresponds to the combinations of outputs at which the vertically integrated firms are producing at constant returns to scale. Any equilibrium combination of outputs produced by vertically integrated firms must lie on this curve. All points on the left hand side of $S P E C$ are points where the vertically integrated firm is producing at product specific decreasing returns to scale. Finally only the points inside the the ellipsis $I N T$ are those where the vertically integrated firm is producing at lower costs than specialized firms. If vertically integrated firms are present in equilibrium, then they must produce combinations of outputs that are in the bold portion of $C R S$.

Figure 1 presents the possible equilibrium outputs produced by the vertically integrated firms for different values of the ratio $\frac{F^{U}}{F^{D}}$. Figure 1a presents the set of equilibrium combination that the vertically integrated firms can choose when the fixed costs of the upstream firm are very high relative to those of the downstream firm; Figure 1b, 1c and 1d present the same set of points for decreasing values of the ratio $\frac{F^{U}}{F^{D}}$. As the ratio $\frac{F^{U}}{F^{D}}$ decreases, the set of combinations at which the vertically integrated firm can be in equilibrium shifts to southeast. The last two cases where the curve INT intersects the $y$-axis (Figures 1c and 1d) occur when $F^{U}+F^{D}+2 \sqrt{F^{U} F^{D}}>2 F^{I} .{ }^{5}$

${ }^{5}$ To see why, observe that when INT and the $y$-axis intersect $k_{s}=0$ and so $2\left(\sqrt{F^{U} w}+\sqrt{F^{D} w}\right)^{2} y^{I}=$ 


\subsubsection{Determination of the Equilibrium Structure}

In the previous section we have determined the set of combination of outputs that vertically integrated firms must produce to exist in equilibrium. The aggregate demands of the intermediate and final goods determine which of the possible combinations vertically integrated firms produce in equilibrium.

The ratio of exogenous aggregate demands, $\frac{K_{s}^{D}}{Y^{D}}$, can be plotted on the same figures 1a and 1b. Depending on where it lies, either on the marked segment or outside of it, a different market structure will occur. To see this, denote by $\frac{K_{s}^{A}}{Y^{A}}$ the line which goes through the point $A$ and by $\frac{K_{s}^{B}}{Y^{B}}$ the line which goes through the point $B$ in figures $2 \mathrm{a}$ and $2 \mathrm{~b}$.

Consider first the case when the output ratio $\frac{K_{s}^{D}}{Y^{D}}$ crosses the marked segment, that is to say $\frac{K_{s}^{A}}{Y^{A}} \leq \frac{K_{s}^{D}}{Y^{D}} \leq \frac{K_{s}^{B}}{Y^{B}}$ in figure $2 \mathrm{a}$ or $0 \leq \frac{K_{s}^{D}}{Y^{D}} \leq \frac{K_{s}^{B}}{Y^{B}}$ in figure $2 \mathrm{~b}$. If each vertically integrated firm chooses to produce a combination of outputs $\left(k_{s}^{I}, y^{I}\right)$ such that $\frac{k_{s}^{I}}{y^{I}}=\frac{K_{s}^{D}}{Y^{D}}$, and such that $n^{I} k_{s}^{I}=K_{s}^{D}$ and $n^{I} y^{I}=Y^{D}$, where $n$ is the number of vertically integrated firms in the market, then there would not be demand left for any specialized firm. Hence only vertically integrated firms would be present in equilibrium. It turns out that this is the only equilibrium market structure when $\frac{K_{s}^{A}}{Y^{A}} \leq \frac{K_{s}^{D}}{Y^{D}} \leq \frac{K_{s}^{B}}{Y^{B}}$ :

Proposition 1 When $\max \left\{0, \frac{K_{s}^{A}}{Y^{A}}\right\} \leq \frac{K_{s}^{D}}{Y^{D}} \leq \frac{K_{s}^{B}}{Y^{B}}$ then only vertically integrated firms are present in equilibrium. Each one of them produces the output combination $\left(y, k_{s}\right)$ which is located where the ratio $\frac{K_{s}^{D}}{Y^{D}}$ crosses the curve CRS. The prices $p^{e}$ and $r^{e}$ are functions of the ratio $\frac{K_{s}^{D}}{Y^{D}}$.

For example, let $\frac{K_{s}^{D}}{Y^{D}}=\frac{3}{2}$ and let the vertically integrated firms choose the output combination $\left(y^{I}, k_{s}^{I}\right)$ such that $k_{s}^{I}=\frac{3}{2} y^{I}$. At that output ratio, $p^{e}=2 w k_{s}^{I}+4 w y^{I}=7 w y^{I}$ and $r^{e}=5 w y^{I}$. In equilibrium firms must make zero profit, hence $y^{I}=\sqrt{\frac{4}{29} \frac{F^{I}}{w}}, p^{e}=7 \sqrt{\frac{4 w}{29} F^{I}}$, $k_{s}^{I}=3 \sqrt{\frac{1}{29} \frac{F^{I}}{w}}$ and $r^{e}=5 \sqrt{\frac{4 w}{29} F^{I}}$.

Vertically integrated firms all choose the same combination of outputs for a given set of input and output prices and they are able to fully serve the aggregate demands $K_{s}^{D}$ and $Y^{D}$. In such context, the upstream and downstream firms cannot be present in the market because they are unable to supply outputs at lower prices (since condition INT holds).

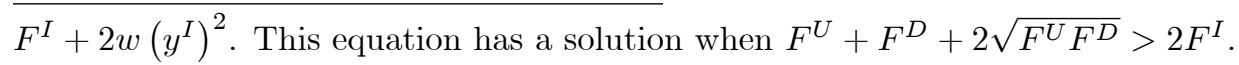


Figure 1a: The Market When Upstream Fixed Costs are Very High

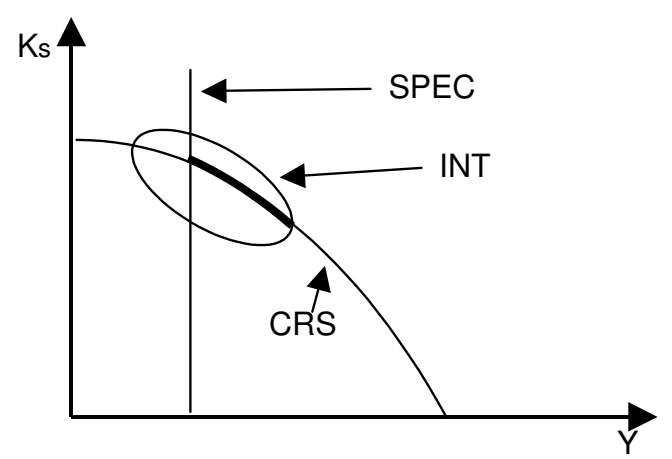

Figure 1b: The Market When Upstream Fixed Costs are High

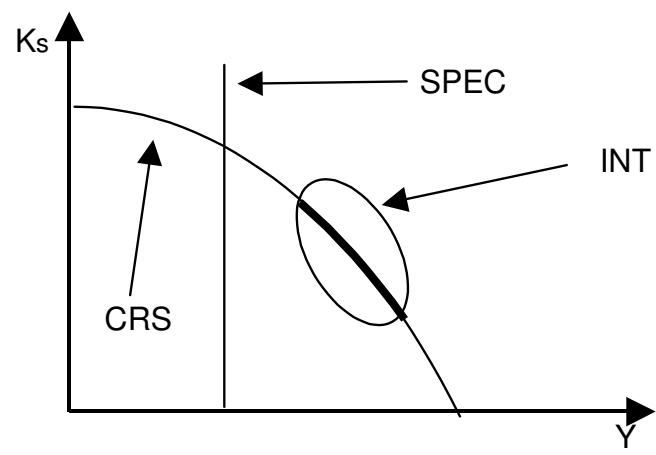

Figure 1c: The Market When Downstream Fixed Costs are High

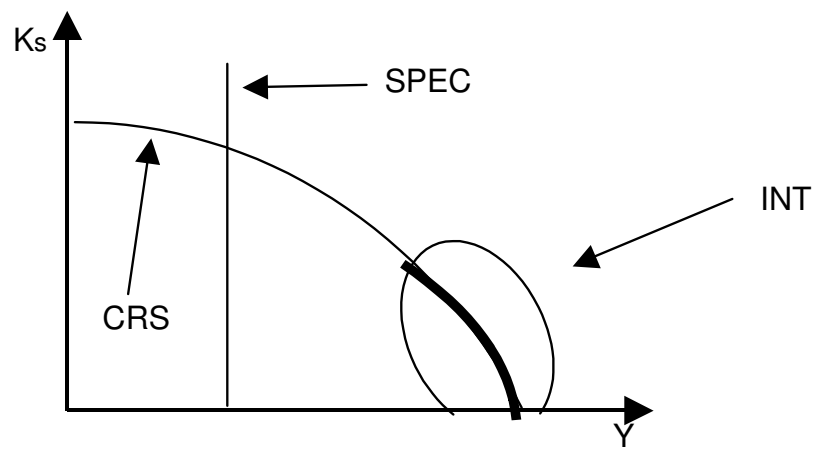

Figure 1d: The Market When Downstream Fixed Costs are Very High

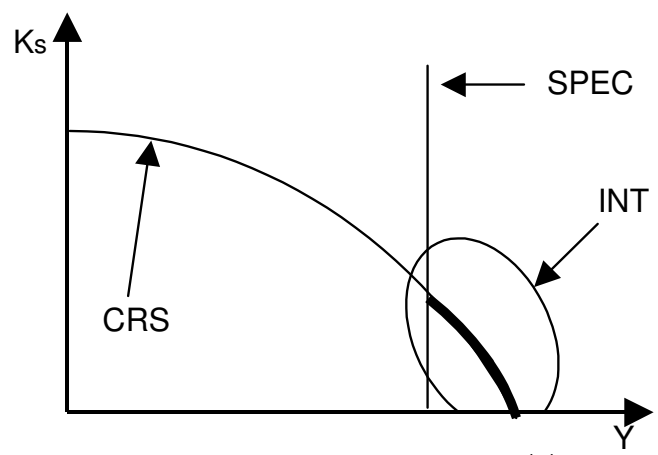


Consider now the case when $\frac{K_{s}^{D}}{Y^{D}}$ crosses the $C R S$ curve outside of the bold segment of the curve $C R S$. We need to address two questions: Will vertically integrated firms be present in equilibrium? Will they be able to serve both the aggregate demand of the final and intermediate outputs? The answer to the first question is always positive (as from Panzar and Willig [1981]) and the answer to the second question is always negative.

Figure 2 presents two sets of possible equilibrium output combinations for the vertically integrated firms, which are drawn for different values of the parameters $F^{U}, F^{I}$, and $F^{D}$. We will only comment on the equilibrium configurations in Figure 2a, since Figure 2b is a special case of Figure 2a.

Suppose that $\frac{K_{s}^{D}}{Y^{D}}>\frac{K_{s}^{B}}{Y^{B}}$, which is the situation when there is a relatively large demand (external to the industry) for $k$. Upstream firms must be present to satisfy part of this demand, since an industry with only vertically integrated firms would be unable to serve the demand. To see this, observe that each vertically integrated firm must choose the same output ratio $\frac{k_{s}^{I}}{y^{I}}$ but $\frac{k_{s}^{I}}{y^{I}} \leq \frac{K_{s}^{B}}{Y^{B}}<\frac{K_{s}^{D}}{Y^{D}}$ so there is no number of integrated firms that can serve both $K_{s}^{D}$ and $Y^{D}$. There is an excess demand of intermediate output that the vertically integrated firms cannot serve in equilibrium. In order for the equilibrium to be perfectly competitive, firms must produce where their average cost are lowest. Hence, the prices of the intermediate input in equilibrium must be $r^{e}=2 \sqrt{F^{U} w}$. Using the condition that the marginal costs must be equal to prices, $2 w\left(y+k_{s}\right)=2 \sqrt{F^{U} w}$ and $2 w\left(y+k_{s}\right)+2 w y=p^{e}$. Using the zero profit condition, we find that the price of the final output in this equilibrium is $p^{e}=2 \sqrt{F^{U} w}+2 \sqrt{w\left(F^{I}-F^{U}\right)}$, which is lower than $2 \sqrt{F^{U} w}+2 \sqrt{w F^{D}}$, the price that a downstream firm would set.

The proofs in this paper use this recursive approach: when specialized firms exist in equilibrium, then they must set prices equal to the lowest average cost. Then, we use the profit maximization condition and the free entry condition to determine the price of the other good that the vertically integrated firms must charge in equilibrium.

The following proposition characterizes the full set of equilibria for this market for different values of the parameters $F^{U}, F^{I}$, and $F^{D}$ :

Proposition 2 There are two general cases.

Case 1) Let $2 F^{U}>F^{I}$. Then: 
1a) If $\frac{K_{s}^{D}}{Y^{D}}>\frac{K_{s}^{B}}{Y^{B}}$, then vertically integrated firms and upstream firms are present in equilibrium. Vertically integrated firms produce a surplus of $k, k_{s}>0$, to be sold in the market. In particular, $r^{e}=2 \sqrt{F^{U} w}$ and $p^{e}=2 \sqrt{F^{U} w}+2 \sqrt{w\left(F^{I}-F^{U}\right)}$.

1b) If $\frac{K_{s}^{D}}{Y^{D}}<\frac{K_{s}^{A}}{Y^{A}}$, then vertically integrated firms and downstream firms are present in equilibrium. Vertically integrated firms produce a surplus of $k, k_{s}>0$, to be sold in the market. In particular, $r^{e}=2 \sqrt{w\left(F^{I}-F^{D}\right)}$ and $p^{e}=2 \sqrt{w\left(F^{I}-F^{D}\right)}+2 \sqrt{F^{D} w}$. Case 2) Let $2 F^{D}>F^{I}$ and $F^{U}+F^{D}+2 \sqrt{F^{U} F^{D}}>2 F^{I}$. Let $\frac{K_{s}^{D}}{Y^{D}}>\frac{K_{s}^{B}}{Y^{B}}$. Then upstream and vertically integrated firms are present in equilibrium. The prices are $r^{e}=2 \sqrt{F^{U} w}$ and $p^{e}=2 \sqrt{w F^{I}}$.

\section{$5 \quad$ Equilibrium with Outsourcing}

In the previous section we have studied the case when vertically integrated firms might produce a surplus of intermediate good. Now we consider the opposite case when they purchase (part of) the intermediate input they need to produce the final output, which occurs when the upstream fixed costs are small relative to the downstream fixed costs. We still maintain that $K_{s}>0$, that is the industry produces a surplus of intermediate outputs that it sells to another industry.

If the size of and $K_{s}^{D} / Y^{D}>K_{s}^{B} / Y^{B}$, then the vertically integrated firm prefers to outsource part of the production to the upstream firm and exploit its economies of vertical scope to produce $y$ at a lower costs than the downstream firm can produce it.

Only the cost function of the vertically integrated firm changes and is as follows:

$$
C^{I_{p}}\left(y, k_{i} ; w, r\right)=F^{I}+w k_{i}^{2}+w y^{2}+r\left(y-k_{i}\right),
$$

where $k_{i}$ represents the amount of intermediate input produced in-house. $y-k_{i}$ is the amount of production of input $k_{i}$ that is outsourced to specialized firms.

We rewrite the cost function in terms of $k_{p}=y-k_{i}$ :

$$
C^{I_{p}}\left(y, y-k_{p} ; w, r\right)=F^{I}+w\left(y-k_{p}\right)^{2}+w y^{2}+r k_{p}
$$


Figure 2a: Equilibrium Outputs of the Vertically Integrated Firm Case I: $\mathrm{F}^{\mathrm{U}}+\mathrm{F}^{\mathrm{D}}+2\left(\mathrm{~F}^{\mathrm{U}} \mathrm{F}^{\mathrm{D}}\right)^{1 / 2}<2 \mathrm{~F}^{\mathrm{I}}$ and $2 \mathrm{~F}^{\mathrm{U}}>\mathrm{F}^{\prime}$

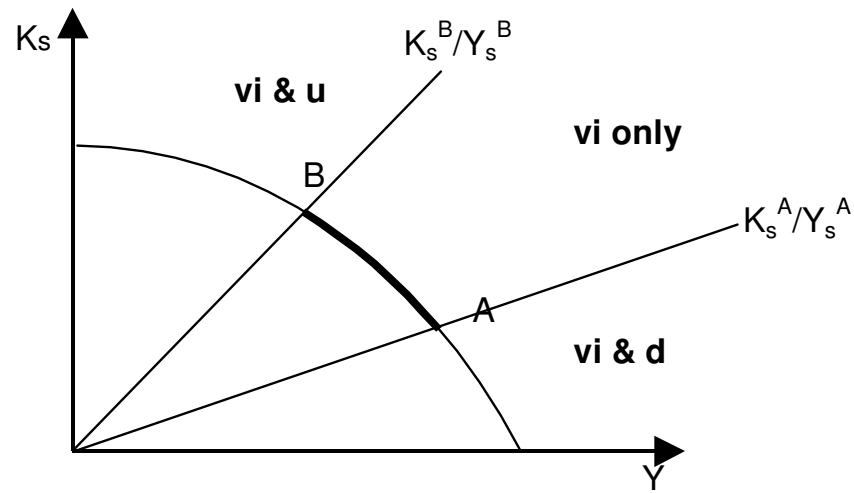

Figure 2b: Equilibrium Outputs of the Vertically Integrated Firm Case II: $F^{U}+F^{D}+2\left(F^{U} F^{D}\right)^{1 / 2}>2 F^{\prime}$ and $2 F^{D}>F^{\prime}$

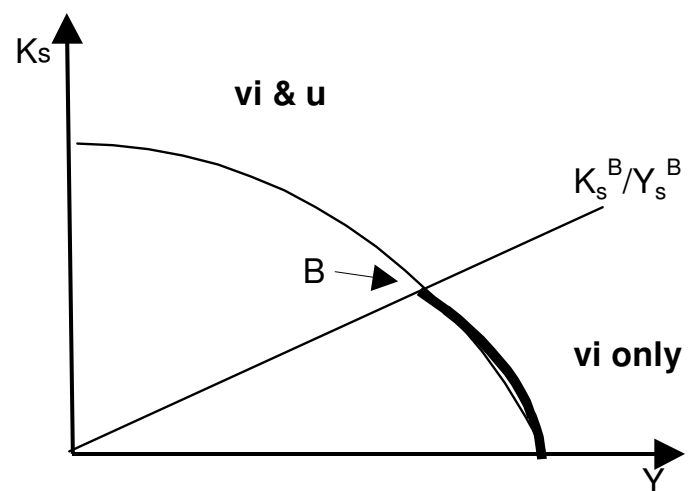


Thus the combinations $\left(k_{p}^{I}, y^{I}\right)$ which are compatible with a vertical competitive equilibrium must be such that:

$$
\begin{gathered}
C R S: F^{I}=w\left(y-k_{p}\right)^{2}+w y^{2}, \\
I N T: 2\left(\sqrt{F^{U} w}+\sqrt{F^{D} w}\right) y \geq F^{I}+w\left(y-k_{p}\right)^{2}+w y^{2}+2 \sqrt{F^{U} w} k_{p} .
\end{gathered}
$$

As before, if vertically integrated firms are present in equilibrium, then they must produce combinations of outputs that are in the bold portion of $C R S$. Also, only the points inside the the ellipsis $I N T$ are those where the vertically integrated firm is producing at lower costs than specialized firms. Notice that the condition that vertically integrated firms produce at product specific decreasing returns to scale $(S P E C)$ is always satisfied when the vertically integrated firm does not produce a surplus of intermediate good.

When vertically integrated firms outsource part of the production of the intermediate input to specialized firms, their internal cost of producing $k$ must equal to the market price for $k$. Hence, the marginal cost of an additional unit of intermediate good must be equal to the market price for it:

$$
O U T: y=k_{p}+\sqrt{F^{U} / w}
$$

For a vertically integrated firm to be in equilibrium, it must produce a combination of outputs that is inside the ellipsis $I N T$, it lies along the curve $C R S$ and is on the line OUT. Figure 3 shows there is only one point that satisfies all three conditions, where OUT intersects $C R S$.

This observation leads to the second set of possible market structures:

Proposition 3 Let $2 F^{D}>F^{I}$ and $F^{U}+F^{D}+2 \sqrt{F^{U} F^{D}}<2 F^{I}$ Then (outsourcing) vertically integrated firms and upstream firms are present in equilibrium. The combination of outputs chosen by the vertically integrated firm is given by $y^{I}=\sqrt{\frac{F^{I}-F^{U}}{w}}$ and $k_{p}^{I}=\sqrt{\frac{F^{I}-F^{U}}{w}}-\sqrt{\frac{F^{U}}{w}}$. The equilibrium prices are given by $r^{e}=2 \sqrt{F^{U} w}$ and $p^{e}=2 \sqrt{w\left(F^{I}-F^{U}\right)}+2 \sqrt{w F^{U}}$.

\section{Equilibrium and Comparative Statics}

In this section, we illustrate the full set of equilibrium market structures for an industry that sells (e.g. "exports") the intermediate good to another industry. To do this, we first give the exact definition of the cost function of the vertically integrated firm: 
Figure 3: Outsourcing

$2 \mathrm{~F}^{\mathrm{D}}>\mathrm{F}^{\mathrm{I}}$ and $\mathrm{F}^{\mathrm{U}}+\mathrm{F}^{\mathrm{D}}+2\left(\mathrm{~F}^{\mathrm{U}} \mathrm{F}^{\mathrm{D}}\right)^{1 / 2}<2 \mathrm{~F}^{\mathrm{I}}$

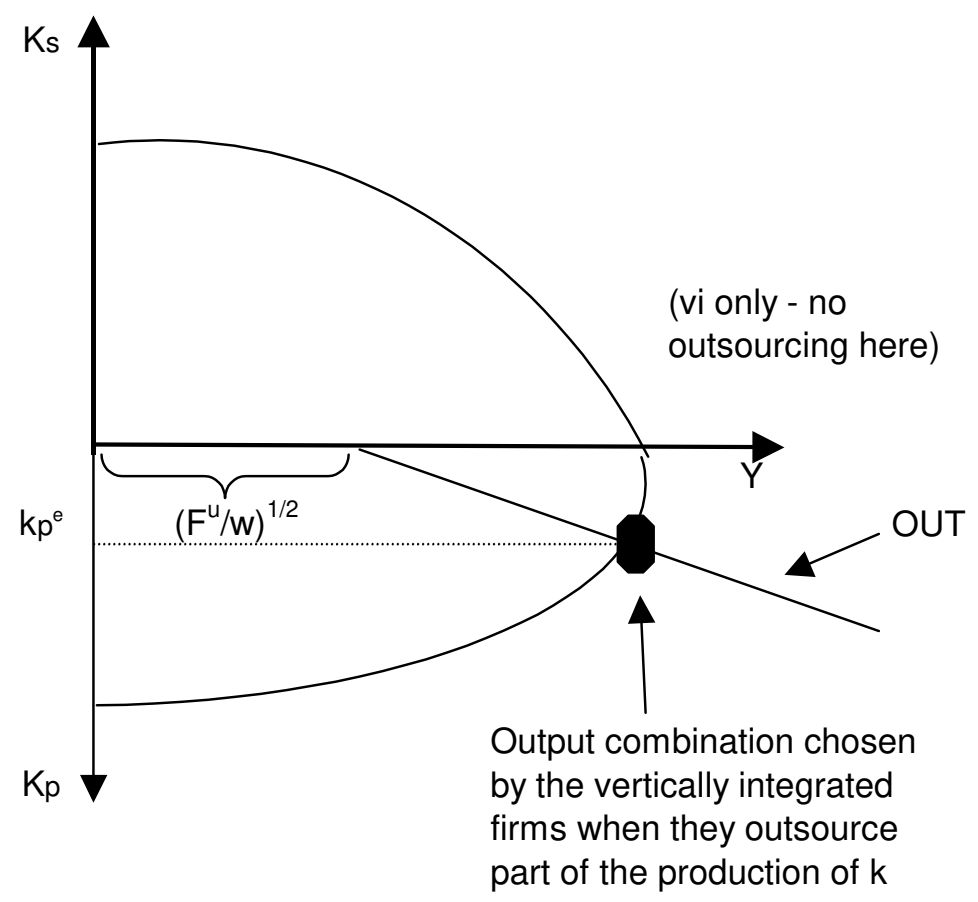

$$
C^{I}=\left\{\begin{array}{c}
C^{I_{s}}\left(y, y+k_{s} ; w, r\right)=F^{I}+w\left(y+k_{s}\right)^{2}+w y^{2}, \text { if } k_{s} \geq 0 . \\
C^{I_{p}}\left(y, y-k_{p} ; w, r\right)=F^{I}+w\left(y-k_{p}\right)^{2}+w y^{2}+r k_{p}, \text { if } k_{p} \geq 0 .
\end{array}\right.
$$

Notice that $C^{I_{s}}\left(y, y+k_{s} ; w, r\right)=C^{I_{p}}\left(y, y-k_{p} ; w, r\right)$ when $k_{s}=0$ and $k_{p}=0$ - the cost function is continuous when the vertically integrated firm consumes all the intermediate output that it produces in-house.

\subsubsection{Equilibria}

Depending on the values taken by $F^{U}, F^{D}$, and $F^{I}$ there are three possible equilibrium configurations for the industry, and they are presented in Figure 4 (where, as usual, $k_{p}=-k_{i}$ for simplicity of exposition). Consider first the cases illustrated by Figure 4a and Figure 4b. Here, there are three possible industry structures: when there is a relatively large demand for the intermediate good, $K_{s}^{D} / Y^{D}>K_{s}^{B} / Y^{B}$, then we have upstream and vertically integrated firms in equilibrium. Vertically integrated firms produce a surplus of intermediate output if the upstream costs are very high while they outsource part of the production of the intermediate to specialized firms if the downstream costs are high. The intuition here is that 
the vertically integrated firms are more flexible than specialized firms and can dedicate their production to the good with the highest costs.

\subsubsection{Comparative Statics}

A Change in the Demand Consider the effect of a change in the aggregate demands. Suppose that we start with $2 F^{D}>F^{I}>2 F^{U}$ and an output ratio $K_{s}^{A} / Y^{A} \leq K_{s}^{D} / Y^{D} \leq$ $K_{s}^{B} / Y^{B}$. We are then in the economy depicted by Figure 4a, where only vertically integrated firms serve the demands $Y^{D}$ and $K_{s}^{D}$. As $K_{s}^{D}$ increases, for example because another industry is growing and demands $k$, then the output ratio $K_{s}^{D} / Y^{D}$ increases as well, and at some point it becomes greater than $\frac{K_{s}^{B}}{Y^{B}}$. As soon as that happens, upstream firms start entering into the market to serve part of the (external) demand of intermediate output. When $K_{s}^{D}$ decreases and becomes smaller than $K_{s}^{A} / Y^{A}$ we will observe entry of downstream firms into the industry. Notice that vertically integrated firms will always be present in the industry as long as economies of vertical scope exist.

A Change in the Costs Now consider changes in the fixed costs. Suppose that both $F^{D}$ and $F^{I}$ increase, while $F^{U}$ remains unchanged, so that we still have $F^{I}<F^{U}+F^{D}$. Suppose, moreover, that $K_{s}^{D} / Y^{D}$ is smaller than $K_{s}^{A} / Y^{A}$ so that we are in Figure 4a, with vertically integrated and downstream firms serving the markets. As $F^{D}$ increases relative to $F^{U}$, the set of combinations that the vertically integrated firms can choose in equilibrium moves to southeast, and when $F^{D}$ becomes larger than $F^{U}$ (with $F^{I}<F^{U}+F^{D}$ still holding), the industry attains the configuration given in Figure 4b, with outsourcing and vertically integrated and upstream firms serving the markets.

\section{Equilibrium and Comparative Statics in an Import- ing Industry}

To close the model, we consider an industry (e.g. its vertically integrated firms) that is a net buyer of the intermediate output, that is $K_{p}=-K_{s}>0$. For this to occur, it has to be true that another industry that produces $k$ can produce it at a lower cost. Call the price of the intermediate good $k$ produced by the other industry as $r^{f}$. Thus, $r^{f}<2 \sqrt{F^{U} w}$, and 
Figure 4a: Equilibrium Market Strucures (I) $2 \mathrm{~F}^{\mathrm{U}}>\mathrm{F}^{\mathrm{I}}>2 \mathrm{~F}^{\mathrm{D}}$

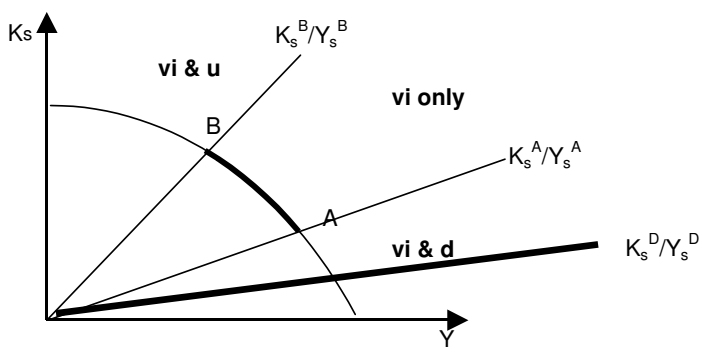

Figure 4b: Equilibrium Market Strucures (II) $2 \mathrm{~F}^{\mathrm{D}}>\mathrm{F}^{\mathrm{l}}$ and $\mathrm{F}^{\mathrm{U}}+\mathrm{F}^{\mathrm{D}}+2\left(\mathrm{~F}^{\mathrm{U}} \mathrm{F}^{\mathrm{D}}\right)^{1 / 2}<2 \mathrm{~F}^{\mathrm{I}}$

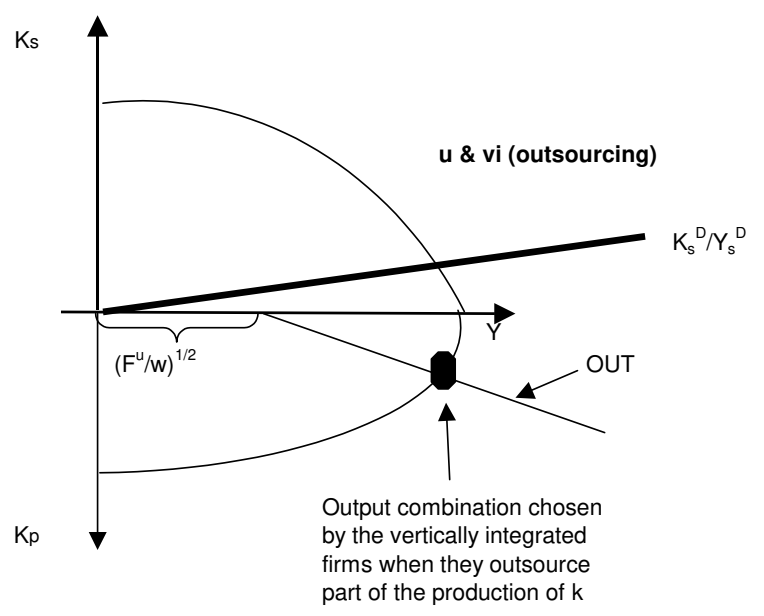


upstream firms cannot be present in equilibrium because their lowest average cost is higher than the market price for the intermediate input.

The relevant issue is which type of firms, between vertically integrated and specialized downstream, exist in equilibrium. Not surprisingly, it depends on the relative magnitude of the fixed costs faced by the downstream and by the vertically integrated firms. When the price of the intermediate good produced by another industry is very low, then vertically integrated firms cannot be in the market, regardless of how strong the economies of vertical scope are. Hence, we will observe downstream specialization when the imported intermediates are very cheap relatively to the prices that the domestic upstream producers would charge.

When the price at which another industry is selling the intermediate good is not too low, then whether we observe vertically integrated firms or downstream specialized firms depends on the magnitude of the economies of vertical scope. If the economies of vertical scope are very strong, then the vertically integrated firms will outsource some of their demand of intermediate good to firms in another industry. However, when the economies of vertical scope are not strong enough, then again only downstream firms will be serving the market for the final good.

The following proposition formalizes these results:

Proposition 4 There are three possible industry configurations:

If $r^{f}>2 \sqrt{w F^{D}}$ and $F^{D}+\frac{\left(r^{f}\right)^{2}}{4 w}-F^{I}<0$, or if $r^{f}<2 \sqrt{w F^{D}}$, then only downstream firms are present in equilibrium, and $p^{e}=r^{f}+2 \sqrt{F^{D} w}$.

If $r^{f}>2 \sqrt{w F^{D}}$ and $F^{D}+\frac{\left(r^{f}\right)^{2}}{4 w}-F^{I}>0$ then only vertically integrated firms are present in equilibrium. The prices are functions of the output ratio $\frac{K_{p}^{D}}{Y^{D}}$.

\section{Summary and Conclusions}

We summarize the results of this paper in Figure 5:

- When the fixed costs for the upstream producers are small relative to those of the downstream producers, vertically integrated firms exploit their economies of vertical scope in the production of the final good, and possibly outsource part of the production of the intermediate input to specialized upstream firms. 
- When the fixed costs for the upstream producers are large relative to those of the downstream producers, then the industry structure is determined by the ratio of the demand for the final good and the demand for the intermediate inputs by another industry.

- Finally, when the industry is a net buyer (e.g. an "importer") of the intermediate input, then vertically integrated firms can only exist in equilibrium if the economies of vertical scope are very strong.

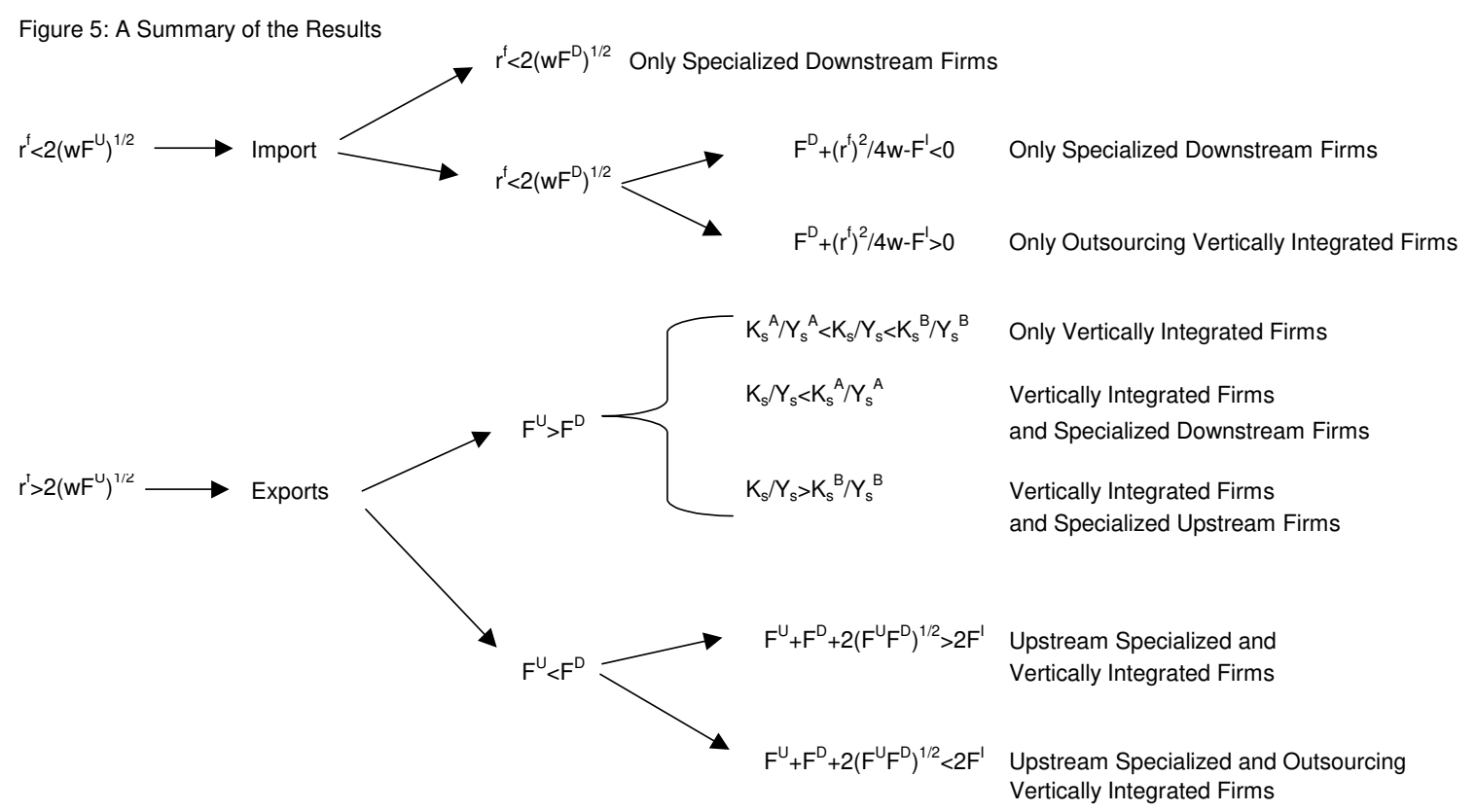

While the results are for specific functional forms, the economic trade-off underlying them is very general and thus the results are likely to hold if we extend the model to allow for cost complementarities or for more general specifications of demand functions. The critical idea is that there must exist some trade-off in the production of the intermediate and final goods. In the model that we have considered, vertically integrated firms can exploit economies of vertical scope in the fixed costs, but face rivalries (rather than complementarities) in the marginal costs. Hence, both types of firms can exist.

Economies of vertical scope in the fixed costs are likely to exist when firms are still learning how to produce final goods and how to design the best intermediate inputs for the final goods. Firms can then benefit from producing both at the same time. As time goes on, 
the process of learning by doing will standardize the production processes, and economies of scope will slowly disappear.

We would like to conclude with a discussion of the limitations of our analysis and with a suggestion for future research topics. First, our paper offers no explanation of the production activities in the industry that buys the surplus of intermediate input. That is, where does the external demand and supply come from for the intermediate good k? Future work might draw interesting conclusions by interacting our ideas of scope economies with those in the trade literature. Second, our paper takes the existence of economies of scope as a black box. Future work might look at what originates economies of scope. For example, one might look at the role of learning-by-doing and investigate how growth affects industry structure or, vice-versa, how endogenizing vertical structure affects growth.

\section{References}

William J. Baumol, John C. Panzar and Robert D. Willig, Contestable Markets and the Theory of Industry Structure, New York, Harcourt Brace Jovanovich, 1982.

Curtis Eaton and S.Q. Lemche, "The Geometry of Supply, Demand, and Competitive Market Structure with Economies of Scope," American Economic Review, Volume 81, Issue 4, September 1991.

Oliver Hart and John Moore, "Incomplete Contracts and Renegotiation," Econometrica, Vol. 56, No. 4 (Jul., 1988), pp. 755-785.

Glenn MacDonald and Alan Slivinski, "The Simple Analytics of Competitive Equilibrium with Multiproduct Firms," American Economic Review, Vol. 77, Issue 5, December 1987.

John C. Panzar and Robert D. Willig, "Economies of Scope," American Economic Review, Vol. 71, No. 2, May 1981, pp. 268-272.

Martin Perry, "Vertical Integration," Handbook of Industrial Organization, 1989, pages $185-255$

Daniel F. Spulber, Regulation and Markets, The MIT Press, 1989.

G. Stigler, "The Division of Labor is Limited by the Extent of the Market," Journal of Political Economy, Vol. 56, N. 3, June 1951, pages 129-141.

Williamson, O., 1985, The Economic Institutions of Capitalism: Firms, Markets and Vertical Contracting, (Free Press, New York). 


\section{Appendix}

\subsection{Proof of Proposition 1}

Proof. Let the equilibrium price of $k$ be $r^{e}$ and the equilibrium price of $y$ be $p^{e}$. Each vertically integrated firm must choose $k^{I}$ and $y^{I}$ such that $C_{y}^{I_{s}}=p^{e}$ and $C_{k}^{I_{s}}=r^{e}$. Because of the assumptions on the cost functions (they are $\mathrm{U}$ shaped) this is a system of two equations in two unknowns with an unique solution (if it exists). Hence, all vertically integrated firms choose the same combination $\left(y^{I}, k_{s}^{I}\right)$ such that $\frac{k_{s}^{I}}{y^{I}}=\frac{K_{s}^{D}}{Y^{D}}$. Thus, $2 w y^{I}\left(1+\frac{K_{s}^{D}}{Y^{D}}\right)=$ $r^{e}$ and $2 w y^{I}\left(2+\frac{K_{s}^{D}}{Y^{D}}\right)=p^{e}$. In equilibrium firms must make zero profit, hence $F^{I}=$ $w\left(y^{I}\right)^{2}\left(1+\left(1+\frac{K_{s}^{D}}{Y^{D}}\right)^{2}\right)$ and so $y^{I}=\sqrt{F^{I} /\left[1+\left(1+\frac{K_{s}^{D}}{Y^{D}}\right)^{2}\right]}$. From $y^{I}$ we can determine $k^{I}$ and the equilibrium prices.

\subsection{Proof of Proposition 2}

Proof. The first case when $2 F^{U}>F^{I}$ is already proved in the text. Consider the second case when $\frac{K_{s}^{D}}{Y^{D}}<\frac{K_{s}^{A}}{Y^{A}}$. Downstream firms must be present in equilibrium because the vertically integrated firms cannot serve all of the demand of $y$ on their own. The price of the final good must then be equal to $p^{e}=r^{e}+2 \sqrt{F^{D} w}$. Hence, $2 w\left(y^{I}+k_{s}^{I}\right)=r^{e}$. This implies that $y^{I}=\sqrt{\frac{F^{D}}{w}}$ and $r^{e}=2 w\left(\sqrt{\frac{F^{D}}{w}}+k_{s}^{I}\right)$. Using the zero profit condition, we find $k_{s}^{I}=\sqrt{\frac{F^{I}-F^{D}}{w}}-\sqrt{\frac{F^{D}}{w}}, r^{e}=2 \sqrt{w\left(F^{I}-F^{D}\right)}, p^{e}=2 \sqrt{w\left(F^{I}-F^{D}\right)}+2 \sqrt{F^{D} w}$. $r^{e}=2 \sqrt{w\left(F^{I}-F^{D}\right)}$ is less than $2 \sqrt{F^{U} w}$, the average cost at the minimum efficient scale of production of the upstream firms.

Now consider the cases when $2 F^{D}>F^{I}$ and $F^{U}+F^{D}+2 \sqrt{F^{U} F^{D}}>2 F^{I} \cdot \frac{K_{s}^{D}}{Y^{D}}>\frac{K_{s}^{B}}{Y^{B}}-$ there is again a relatively large demand for $k$ and upstream firms must be present to satisfy at least part of this demand. Vertically integrated firms are still present. To see this, first observe that $r^{e}=2 \sqrt{F^{U} w}$ since upstream firms must be in equilibrium. The only way in which the optimization problem of the vertically integrated firm could have an interior solution is if $k_{s}^{I}=\sqrt{\frac{F^{U}}{w}}-\sqrt{\frac{F^{I}-F^{U}}{w}}$ which is negative. We assumed that the vertically integrated firm cannot purchase intermediate input, hence the best that the vertically integrated firm can do is to set $k_{s}^{I}=0$. Then, using $C_{y}^{I_{s}}=p^{e}$ and $k_{s}^{I}=0$, we find $4 w y=p^{e}$. Using the CRS condition we have $F^{I}=2 w y^{2}, y=\sqrt{\frac{F^{I}}{2 w}}$. Thus, $p^{e}=2 \sqrt{w F^{I}}$, which is smaller than 
the price that the downstream firm would set, $2 \sqrt{F^{U} w}+2 \sqrt{F^{D} w}$.

\subsection{Proof of Proposition 3}

Proof. To show that a market structure with vertically integrated and upstream firms would be an equilibrium configuration, observe that the price of the intermediate input has to be equal to $r=2 \sqrt{F^{U} w}$. The vertically integrated firm chooses $k_{i}^{I}$ and $y^{I}$ to maximize its profits. Thus, $y^{I}=\sqrt{\frac{F^{I}-F^{U}}{w}}$ and $k_{i}=\sqrt{\frac{F^{U}}{w}}$, or $k_{p}^{I}=\sqrt{\frac{F^{I}-F^{U}}{w}}-\sqrt{\frac{F^{U}}{w}}$. The price of the final output is then $p^{e}=2 \sqrt{w\left(F^{I}-F^{U}\right)}+2 \sqrt{w F^{U}}$, lower than the price the downstream firms would be able to set. The profits of both types of firms are zero.

\subsection{Proof of Proposition 4}

Proof. Suppose that downstream firms are in present in equilibrium and vertically integrated firms are not outsourcing any of the production of $k$. Then $2 w\left(y^{I}+k_{s}^{I}\right)=r^{f}$ and $2 w\left(y^{I}+k_{s}^{I}\right)+2 w y^{I}=r^{f}+2 \sqrt{F^{D} w}$. Then $y^{I}=\sqrt{\frac{F^{D}}{w}}$ and $k_{s}^{I}=\frac{r^{f}}{2 w}-y^{I}$ or $k_{s}^{I}=\frac{r^{f}}{2 w}-\sqrt{\frac{F^{D}}{w}}$. This can be an equilibrium if $r^{f}>2 \sqrt{w F^{D}}$. However, it is not an equilibrium because the vertically integrated firm makes positive profits. To see this, observe that the profit can be written as $\left(2 \sqrt{w F^{D}}+r^{f}\right) \sqrt{\frac{F^{D}}{w}}+r^{f}\left(\frac{r^{f}}{2 w}-\sqrt{\frac{F^{D}}{w}}\right)-F^{I}-w\left(\frac{r^{f}}{2 w}\right)^{2}-F^{D}$, which turns out to be equal to $F^{D}+\frac{\left(r^{f}\right)^{2}}{4 w}-F^{I}$. Only downstream firms are present in equilibrium if $F^{D}+\frac{\left(r^{f}\right)^{2}}{4 w}-F^{I}<0$, while only vertically integrated firms are present in equilibrium if $F^{D}+\frac{\left(r^{f}\right)^{2}}{4 w}-F^{I}>0$.

Now consider $r^{f}<2 \sqrt{w F^{D}}$. If vertically integrated firms and downstream firms are in equilibrium, then $2 w k_{i}^{I}-r^{f}=r^{f}$ and $2 w y^{I}+r^{f}=r^{f}+2 \sqrt{F^{D} w}$, with $y^{I}=\sqrt{\frac{F^{D}}{w}}$ and $k_{p}^{I}=\sqrt{\frac{F^{D}}{w}}-\frac{r^{f}}{2 w}$. The profit of the vertically integrated firm is now given by $F^{D}-F^{I}-\frac{\left(r^{f}\right)^{2}}{2 w}$ which is again less than zero, and so only downstream firms are present in equilibrium. 